\title{
Obstructive Salivary Gland Disease and Sialendoscopy
}

\author{
Prithvi S. Bachalli and Aditya Moorthy
}

\subsection{Introduction}

Salivary gland diseases have been described in the literature for centuries. From the time of Hippocrates to the descriptions of parotid tumours in the sixteenth century and the anatomical descriptions of the ductal systems of the major salivary glands in the seventeenth century, we have assimilated knowledge of anatomy and pathology of these glands [1].

The conventional approach to infections of the salivary glands is medical management, occasional expression of the stone through the papilla, marsupialisation of the duct following removal of sialolith. Failing all, removal of the involved gland [2].

Minimally invasive surgery especially those in which endoscopes are utilized has gradually become popular over the last three decades. As a whole, the trend in surgery is to improve function and hasten recovery. To achieve these ends, a combination of technology and smaller incisions has been crucial. Hence there is an increasing interest to manage salivary gland diseases endoscopically [3].

Sialendoscopy is the endoscopic management of obstructive salivary ductal disease and has rapidly become the procedure of choice for such conditions.

\subsection{Obstructive Salivary Diseases}

Salivary gland diseases can broadly be classified into ductal and parenchymal disorders.

Electronic Supplementary Material The online version of this chapter (https://doi.org/10.1007/978-981-15-1346-6_47) contains supplementary material, which is available to authorized users.

P. S. Bachalli $(\varangle) \cdot$ A. Moorthy

Department of Oral and Maxillofacial Surgery, Rangadore

Memorial Hospital, Apollo Hospitals,

Bangalore, Karnataka, India
Some conditions, such as Sjögren's syndrome and juvenile recurrent parotitis, tend to have an overlapping component and thus are difficult to segregate.

Ductal obstructions can be commonly attributed to-

- Sialoliths,

- Strictures within the duct or at the punctum,

- Presence of mucous plugs, and

- Retrograde bacterial infection.

The majority of obstructive salivary gland disease can be attributed to the presence of stones or sialoliths. Almost $60-70 \%$ of reported obstructive salivary disease comprises of sialolithiasis [4], which occurs in about $1.2 \%$ of the population. Amongst the major salivary glands, the submandibular is most affected (87\%), then the parotid (10\%), and the sublingual (3\%) to a much lesser extent [5].

Although sialolithiasis has been reported as the most common cause of salivary ductal obstructions, strictures, mucous plugs and the rare foreign body can cause significant obstruction to ductal flow.

\subsection{Sialendoscopy}

Sialendoscopy is a minimally invasive technique which makes use of miniature endoscopes to diagnose and treat salivary gland pathologies, including sialolithiasis, sialadenitis and strictures.

It is fast becoming the investigating procedure of choice for such conditions. Sialendoscopy has grown as a subspeciality in the last three decades, since the first attempts to retrieve salivary stones endoscopically were carried out. It is slowly gaining popularity and awareness in the last decade and a half. 


\subsection{Evolution of Sialendoscopy}

It took a few centuries, after the discovery of the ductal systems, for the first endoscopy to be reported. In 1990, Konigsberger et al. reported the first successful salivary endoscopy using a 0.8 - mm flexible endoscope. Katz removed a stone with a flexible scope using blind passage of a basket in 1991, and for over a decade, both Nahlieli et al. and Marchal described various types of sialendoscopy instruments and approaches [3].

The last decade and a half has witnessed significant development of minimally invasive techniques for diagnosing and treating salivary ductal obstructions. Like with other surgical fields, this has led to a paradigm shift from open procedures to minimally invasive and endoscopic techniques with the emphasis on gland preservation and restoration of function. The miniaturization of endoscopes and advancing technology have made exploration and visualization of salivary ductal system possible using sialendoscopes [4].

\subsection{Indications and Contraindications}

As mentioned previously, sialolithiasis is the most common obstructive condition affecting the salivary duct and therefore is where sialendoscopic/sialendoscopy-assisted retrieval of sialoliths is most indicated.

Ductal strictures which may be secondary to calculi or those at the papilla opening can be very effectively treated by serial dilatation with sialendoscopes of increasing diameter [4].

Juvenile recurrent parotitis (JRP) is an inflammatory condition affecting the parotid gland and is the second most common condition in children after mumps [6, 7]. The condition affects the paediatric population primarily especially the ages between 3 and 6 years and occasionally persists in adolescence [8]. As the cause is unknown, management has generally been conservative. Anatomical aberrations, such as kinks in the duct, dehydration and possible ascending bacterial infection, have been hypothesised as causes.

Dilatation and lavage as a consequence of performing sialendoscopy has provided relief from symptoms and reduction in the frequency of attacks, even though the mechanism is not fully understood. This condition has a tendency of spontaneous cessation at puberty.

Although in Sjogren's syndrome, where the parenchyma of the parotid gland is primarily affected, there is also an associated ductal pathology, namely, strictures. Here too dilatation via sialendoscopy can provide symptomatic relief.

Acute inflammation of salivary glands and ducts is a contraindication to performing sialendoscopy. The duct is essentially a condensation of surrounding epithelium and not a rigid structure. In such an inflamed state, the risk of creating a false passage or perforating the duct is significantly higher [4].

Management in such situations is conservative, mainly with the use of appropriate antibiotics and analgesics, incision and drainage of a collection, and removal of cause, such as a sialolith, if easily visible or accessible, to allow acute symptoms to settle before a formal sialendoscopy is carried out.

Trismus is a relative contraindication to performing sialendoscopy, as naturally, reduction in mouth opening makes it both difficult to introduce and manoeuvre the scopes.

\subsection{Investigations}

A simple ultrasound in the hands of an experienced sonologist usually provides adequate information prior to sialendoscopy. It is a non-invasive, economical investigation which can determine the presence and size of sialoliths, strictures, dilatation or fibrosis of the duct. Alternatively a CT scan can be considered to achieve the same result.

MR sialograms are particularly useful in identifying strictures or areas of stenosis. However, like conventional sialography the papilla needs to be dilated and cannulated to inject the dye. In the hands of an inexperienced operator, damage to papilla might make performing sialendoscopy impossible.

\subsection{Armamentarium}

The sialendoscope can be divided very simply into two systems: the Marchal All-in-one (Fig. 47.1) and the Modular system (Fig. 47.2).

As the name suggests, the all-in-one has an irrigation port, a working channel through which various instruments can be introduced, and fibre optics in the same unit. This scope serves as both diagnostic and as an interventional tool.

The modular sialoendoscope consists only of a telescope, attached fibre-optic cable and an eyepiece. Interchangeable sheaths of various diameters are available which fit onto the basic telescope [6]. This scope with the appropriate sheaths can be used for both diagnostic and interventional purposes.

The sheaths used in the modular system make it rigid and unfortunately also bulky; thus significant dilatation of the papilla is required to introduce the scope [6].

Unlike other endoscopes, sialendoscopes come with only a zero-degree viewing angle. The all-in-one is semirigid, with a 5-15-degree angulation at the tip to facilitate manoeuvrability especially while exploring branches of the duct.

Diagnostic scopes come with only an irrigation port, whereas a working channel is also provided in therapeutic scopes to introduce instruments such as wire baskets and graspers for entrapping stones, balloons for stricture dilation 


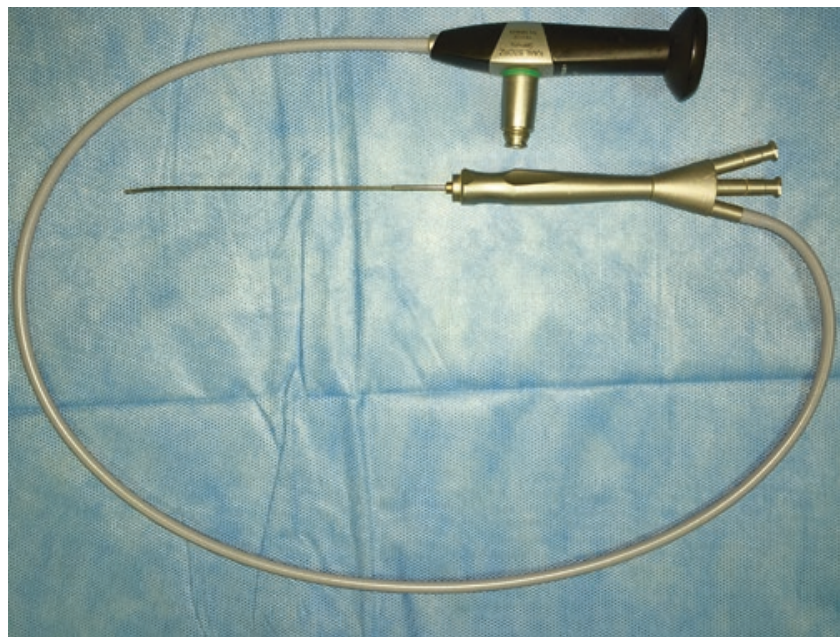

CAssociation of Oral and Maxillofacial Surgeons of India

Fig. 47.1 Marchal All-in-one sialendoscope

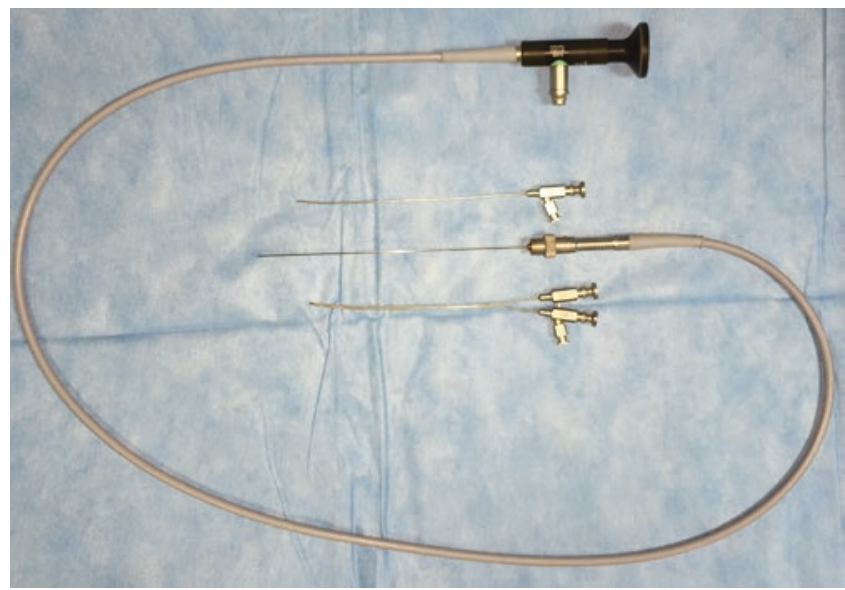

CAssociation of Oral and Maxillofacial Surgeons of India

Fig. 47.2 Modular sialendoscope with sheaths

and a holmium laser fibre or a micro drill to fragment sialoliths [6].

Diameter of available scopes range from 0.9 to $1.6 \mathrm{~mm}$, the smallest purely diagnostic and others having various sizes of the working channel to allow certain instruments only. This is a disadvantage of the all-in-one system in that the entire scope has to be changed if a larger instrument is required, whereas in the modular only the sheath can be changed [6].

Preliminary instruments, those that are used to dilate the papilla to facilitate the introduction of the scope, are also available. These include two types of dilators, papillotomy scissors, guide wires and hollow dilators [6] (Fig. 47.3).

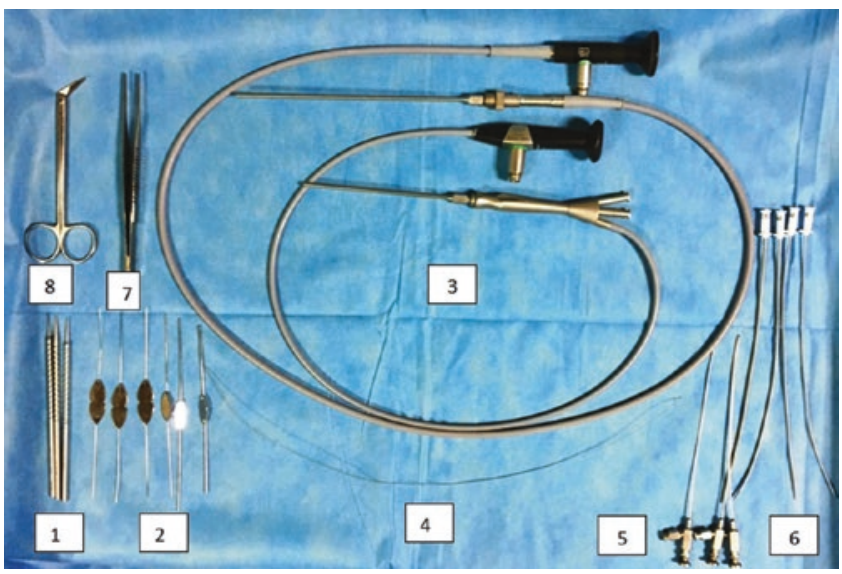

CAssociation of Oral and Maxillofacial Surgeons of India

Fig.47.3 Armamentarium. (1) Conic dilators, (2) Lacrimal probes, (3) Sialendoscopes, (4) Guide wire, (5) Sheaths for Modular sialendoscope, (6) hollow dilators, (7) vascular forceps, (8) papillotomy scissors

\subsection{Anaesthesia and Technique}

Anaesthetic of choice depends on the clinical and radiologically findings and the anticipated difficulty of the procedure. Local anaesthetic, with or without sedation, is usually adequate for a diagnostic procedure. In cases where sialolith retrieval is planned, either endoscopically or with a combined approach, general anaesthetic is usually preferable. Antisecretory agents like atropine and glycopyrrolate are avoided [6].

\subsubsection{Positioning}

Patient is placed supine with head fixed on a head rest and turned towards the surgeon. Shoulder extension is preferable. The monitor is placed opposite the surgeon. The assistant is next to the surgeon [6].

\subsubsection{Identification, Cannulation and Dilatation of Punctum}

The Wharton's duct punctum is comparatively more difficult to locate given the position is quite variable. There may even be a 'hood' of tissue obscuring the opening. In such situations, local anaesthetic can be infiltrated around the area which stiffens the opening, thereby aiding in location. Occasionally an incision might need to be placed on the floor of the mouth accompanied by minimal dissection to locate the duct. The Stensen's duct opening is far easier to identify. It is located on the buccal mucosa opposite upper second molar. Once the punctum is identified, the opening is serially dilated with conical dilator and probes of various sizes [6]. 


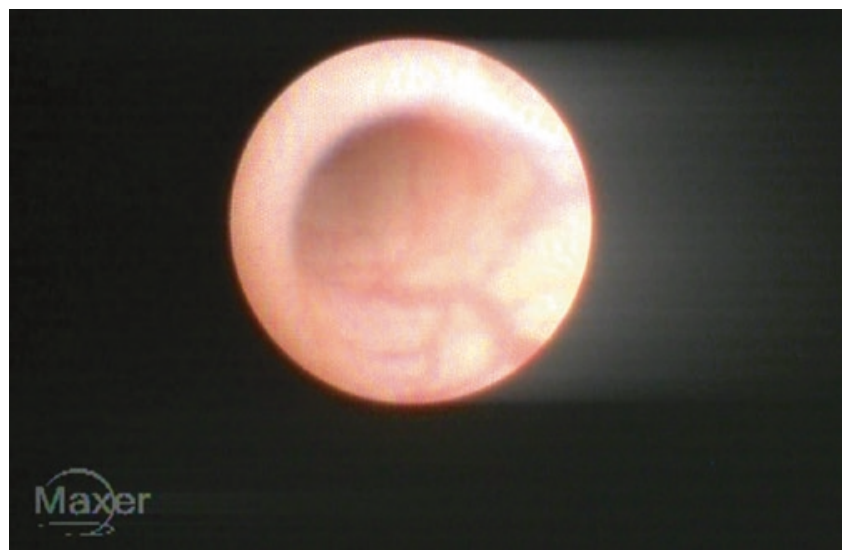

CAssociation of Oral and Maxillofacial Surgeons of India

Fig. 47.4 Normal duct with vascular markings

\subsubsection{Sialendoscopic Evaluation}

Once the punctum is adequately dilated, the appropriate sialendoscope is introduced into the duct. The duct is normally a collapsed structure, and irrigation fluid is required to keep the lumen open to visualise the duct and to advance the scope (Fig. 47.4). Normal saline is the preferred choice of irrigant solution and is diluted with local anaesthetic if the patient is awake for the procedure.

The irrigant also serves an additional purpose as this also performs lavage of the duct, thereby washing out debris and mucous plugs which might be accumulated [6].

The endoscope is slowly and gently passed from the punctum till branches are identified and explored if possible. This point of division of the duct is known as the hilum and is considered the end point of sialendoscopy. Alternatively, the presence of pathology such as a sialolith or stricture may prevent complete exploration of the duct (Figs. 47.5 and 47.6). It is important to note that once the pathology has been treated, the entire duct must be explored so as to ensure there are no further causes of obstruction, including the withdrawal of the scope under vision [6].

As the scope is guided through the primary and secondary ducts, the colour and texture of the mucosa are noted which are an indicator of the presence of inflammation. In relation to sialoliths, the size, shape and position need to be assessed as these will determine the method of retrieval [6].

Between the submandibular and the parotid duct, the Stensen's duct is comparatively more difficult to navigate due to the presence of the masseteric bend.

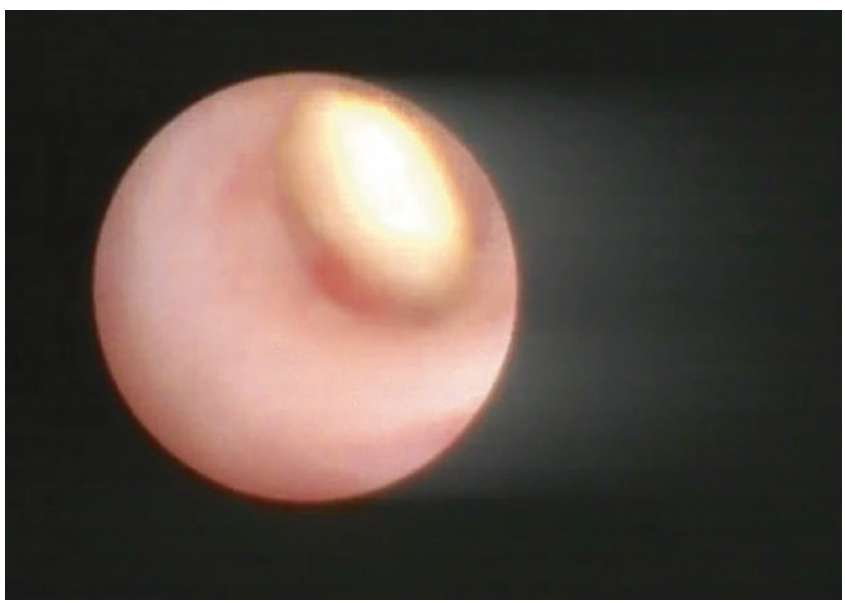

CAssociation of Oral and Maxillofacial Surgeons of India

Fig. 47.5 Sialolith

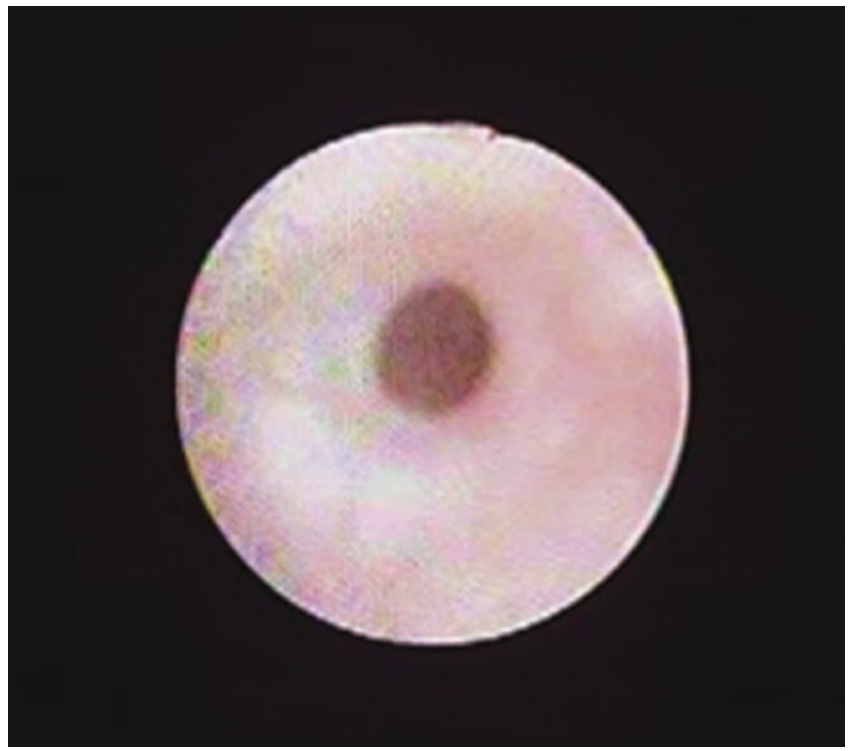

CAssociation of Oral and Maxillofacial Surgeons of India

Fig. 47.6 Stricture

\subsection{Diagnostic vs. Interventional Sialendoscopy}

Indications for a diagnostic procedure include clinical symptoms, yet an unremarkable ultrasound and stricture at the papilla opening, to assess size and position of sialoliths.

Diagnostic sialendoscopy is a low morbidity, minimally invasive technique, which becomes the investigational procedure of choice for salivary duct pathologies in all age 
groups. Sialendoscopy has the advantage of offering a realtime ductal view. Diagnostic endoscopy is occasionally therapeutic, in minor obstructions caused by mucous plugs which can be relieved by lavage.

Interventional sialendoscopy makes use of a wide array of armamentarium including wire baskets (Fig. 47.7), balloon catheters and holmium laser to assist in sialolith retrieval (Fig. 47.8).

Sialoliths with sizes ranging between 3 and $4 \mathrm{~mm}$ are amenable for endoscopic retrieval. Here they may be

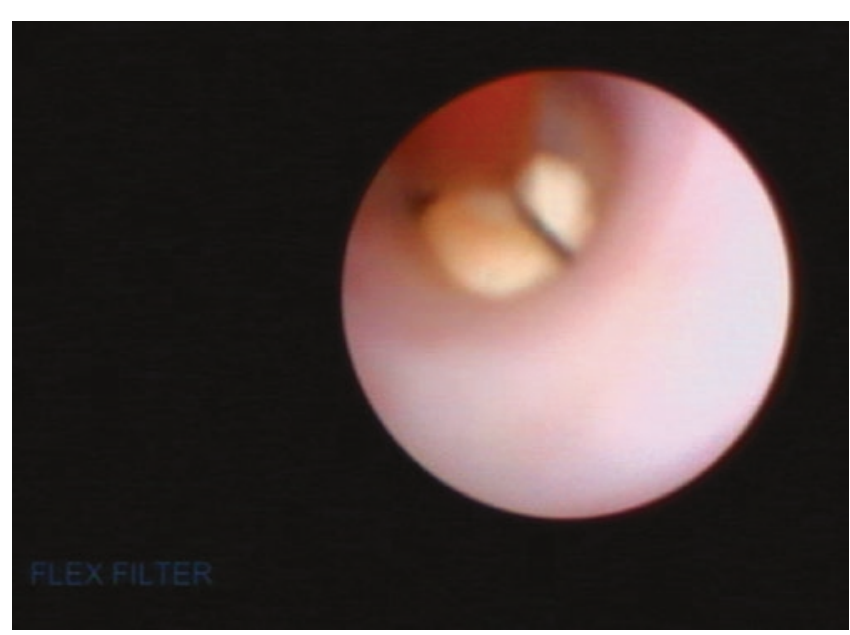

CAssociation of Oral and Maxillofacial Surgeons of India

Fig. 47.7 Sialolith entrapped within wire basket

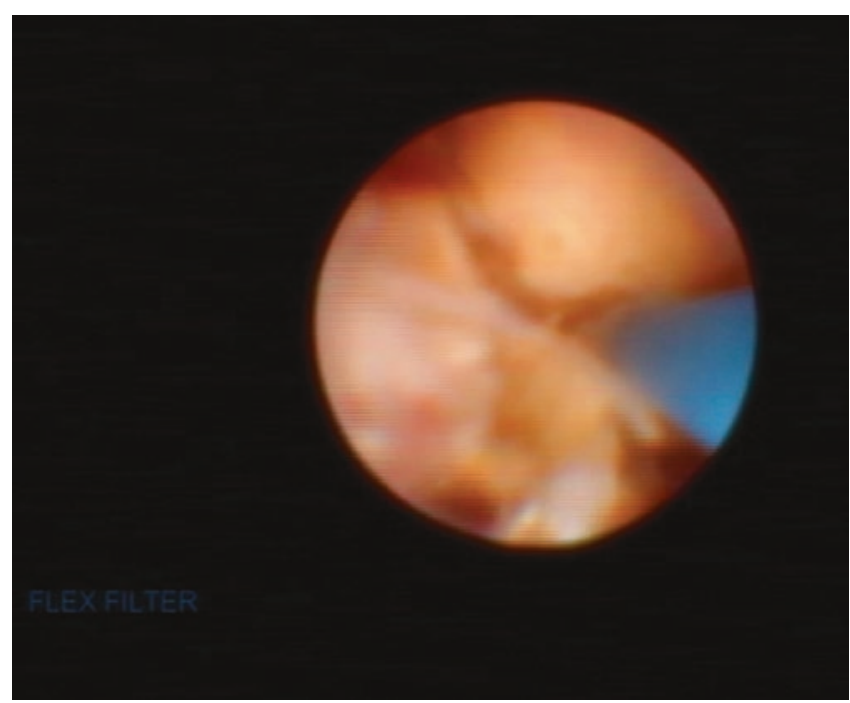

CAssociation of Oral and Maxillofacial Surgeons of India

Fig. 47.8 Laser lithotripsy entrapped and retrieved by use of a wire basket or dragged to the punctual opening where a small incision is placed to facilitate removal.

Larger stones require a combined approach, whereby the position of the sialolith is identified and marked endoscopically and retrieved with an open surgical approach. The open approaches for submandibular sialoliths may be as small as a papillotomy or an extensive dissection of the floor of the mouth. The lingual nerve and iatrogenic ranula formation are important considerations when operating in this region. Large parotid stones require a SMAS flap, for the identification and protection of facial nerve branches and the duct, as well as reconstruction post-retrieval. Sialocele formation is an important consideration.

Hollow bougies of increasing diameter can threaded over a guide wire to widen strictures at the papillae. Balloons are another means of treating ductal strictures and once introduced through the working channel of the sialendoscope are inflated to dilate narrowed areas of the duct wall.

Stents are placed to maintain the patency of the duct and also to allow an incised duct to heal over with the aim of preventing stenosis. These stents are generally kept in place for up to 3 weeks. The authors have devised a salivary stent (Moorthy-Bachalli stent), made of polyurethane. These stents are available in sizes ranging from 3.5 French (diameter, $1 \mathrm{Fr}=0.3 \mathrm{~mm}$ ) to 8 French and lengths from 5 to $7 \mathrm{~cm}$ with markings every centimetre. The stents also have a flange with prefabricated suture holes to facilitate suturing to the surrounding mucosa. An Ethilon 4-0 suture is preferred by the authors.

\subsection{Complications}

Like any other surgical procedure, open or endoscopic, sialendoscopy has its fair share of complications (Table 47.1).

Table 47.1 Complications of sialendoscopy

\begin{tabular}{l|l}
$\begin{array}{l}\text { Complications } \\
\text { Inability to locate } \\
\text { papilla }\end{array}$ & $\begin{array}{l}\text { Abort procedure/open approach to } \\
\text { submandibular/parotid duct }\end{array}$ \\
\hline False passage & $\begin{array}{l}\text { If possible to locate duct-proceed, and abort } \\
\text { procedure otherwise }\end{array}$ \\
\hline Duct perforation & $\begin{array}{l}\text { If possible to navigate beyond-proceed } \\
\text { In case of floor of mouth or cheek oedema, } \\
\text { then abort }\end{array}$ \\
\hline Duct avulsion & Gland excision
\end{tabular}




\subsection{Summary}

When compared to other medical specialities, particularly otorhinolaryngologists, maxillofacial surgery is still in a primitive stage as far as the use of endovision is concerned. Hopefully an interest in areas like endoscopic management of salivary gland and temporomandibular joint diseases will narrow this gap.

Sialendoscopy is still in its infancy, in many parts of the world. Despite the challenges, growing awareness and the added attraction of minimally invasive surgery is steadily making it a popular choice for managing salivary ductal disease.

\section{References}

1. Marchal F. Sialendoscopy: the endoscopic approach to salivary gland ductal pathologies. Endo Press: Tuttlingen; 2012. pp 8.
2. Al-Abri R, Marchal F. New era of endoscopic approach for sialolithiasis: sialendoscopy. SQU Med J. 2010;10(3):382-7. Epub 14th Nov 10.

3. Erkul E, Gillespie MB. Sialendoscopy for non-stone disorders. Laryngoscope Investig Otolaryngol. 2016;1:140-5.

4. Singh PP, Gupta N, Goyal A, Tomar S. Interventional sialendoscopy for parotid ductal calculi: our preliminary experience. Indian J Otolaryngol Head Neck Surg. 2012;64(3):252-6.

5. Kopeć T, Szyfte W, Wierzbicka M. Sialoendoscopy and combined approach for the management of salivary gland stones. Eur Arch Otorhinolaryngol. 2013;270:219-23.

6. Singh PP, Gupta V. Sialendoscopy: introduction, indications and technique. Indian J Otolaryngol Head Neck Surg. 2014;66(1):74-8.

7. Harold D. Baurmash: chronic recurrent parotitis: a closer look at its origin, diagnosis, and management. J Oral Maxillofac Surg. 2004;62:1010-8.

8. Canzi P, Occhini A, Pagella F, Marchal F, Benazzo M. Sialendoscopy in juvenile recurrent parotitis: a review of the literature. Acta Otorhinolaryngol Ital. 2013;33:367-73.

Open Access This chapter is licensed under the terms of the Creative Commons Attribution 4.0 International License (http://creativecommons. org/licenses/by/4.0/), which permits use, sharing, adaptation, distribution and reproduction in any medium or format, as long as you give appropriate credit to the original author(s) and the source, provide a link to the Creative Commons license and indicate if changes were made.

The images or other third party material in this chapter are included in the chapter's Creative Commons license, unless indicated otherwise in a credit line to the material. If material is not included in the chapter's Creative Commons license and your intended use is not permitted by statutory regulation or exceeds the permitted use, you will need to obtain permission directly from the copyright holder. 